\title{
Comparative Analysis of Channel Estimation Techniques in SISO, MISO and MIMO Systems
}

\author{
Okoyeigbo Obinna, Okokpujie Kennedy, Omoruyi Osemwegie, and Nkordeh Nsikan
}

\begin{abstract}
The ever-growing need for high data rate, bandwidth efficiency, reliability, less complexity and less power consumption in our communication systems is on the increase. Modern techniques have to be developed and put in place to meet these requirements. Research has shown, that compared to conventional Single Input Single Output (SISO) systems, MultipleInput Single Output (MISO), and Multiple-Input MultipleOutput (MIMO) can actually increase the data rate of a communication system, without actually requiring more transmit power or bandwidth. This paper aims at the investigation of the existing channel estimation techniques. Based on the pilot arrangement, the block type and comb type are compared, employing the Least Square estimation (L.S) and Minimum Mean Squared Error (MMSE) estimators. Pilots occupy bandwidth, minimizing the number of pilots used to estimate the channel, in order to allow for more bandwidth utilization for data transmission, without compromising the accuracy of the estimates is taken into consideration. Various channel interpolation techniques and pilot-data insertion ratio are investigated, simulated and compared, to determine the best performance technique with less complexity and minimum power consumption. As performance measures, the Mean Squared Error (MSE) and Bit Error Rate (BER) as a function of Signal to Noise power Ratio (SNR) of the different channel estimation techniques are plotted, in order to identify the technique with the most optimal performance. The complexity and energy efficiency of the techniques are also investigated. The system modelling and simulations are carried out using Matlab simulation package. The MIMO gives the optimum performance, followed by the MISO and SISO. This is as a result of the diversity and multiplexing gain experienced in the multiple antenna techniques using the STBC.
\end{abstract}

Keywords-Multiple-input multiple-output (MIMO), Multipleinput Single output (MISO), Single input Single output (SISO), Least Square estimation (L.S), Minimum Mean Squared Error (MMSE) estimators, Mean squared error (MSE) and Bit error rate $(B E R)$

\section{INTRODUCTION}

$\mathbf{T}$ HE communication needs of man in terms of high data rate, wider coverage, reliable and energy efficient communication systems are ever growing. The available communication resources in terms of bandwidth, power and cost, required to meet these ever growing needs are limited. As a result, it is of utmost importance to utilize these limited resources to satisfy our unlimited communication needs. The earth is our home, therefore its conservation and sustainability is our responsibility. Research has shown the adverse effects of global warming on the environment. It is also said that the amount of $\mathrm{CO}_{2}$ produced by the Information and Communication Technology (ICT) industry indirectly is comparable to that of the aviation industry. The ICT industry is also said to consume about two percent of the global energy supply. Due to the expected increase in data rate, energy consumption is expected to double by 2020 . Hence, the need for the Green Communication consortium which is focused on energy efficiency with high data rate communication systems [1]. The wireless communication channel, which is an important part of wireless communication systems, poses a great challenge. This is due to its frequency selectivity, time varying and random nature, which makes it difficult to be engineered [2]. This can be combated with proper equalization techniques. But the knowledge of the channel is required for equalization, and in reality, it is unavailable at the receiver, hence the need for channel estimation [3,4]. With techniques like MIMO and channel estimation, this random nature of the channel can be exploited. The effects of the wireless channel on the signals that propagate through it could be constructive or destructive. The multiple propagation paths that exist in a wireless environment and the interference of symbols and channels are also an issue. Also with the increase in number of mobile communication system users, some measures need to be put in place to accommodate these users within the limited available spectrum, without interference. One of such techniques is the OFDM, which maximizes bandwidth and overcomes ISI [5, 6]. By carrying out channel estimation in MIMO-OFDM systems, high data rates, wider coverage, system reliability, spectral and energy efficiency can be achieved $[7,8,9,10]$. The aim of this paper is to study and investigate the wireless communication channel, and different channel estimation techniques employed in SISO, MISO and MIMO-OFDM systems, to model and simulate a wireless communication system which employs these studied channel estimation techniques, and to compare their performance in terms of accuracy of the channel estimates, accuracy of the detected signal using the channel estimates for equalization, and finally to identify the techniques with optimum performance, less complexity and less power consumption. Hence, meeting the requirements of the Green Communications Project [1]. This paper is modelling and simulation based. Therefore the systems was modelled and represented using mathematical representation, and these mathematical models was simulated using a software package. The software simulation tool employed is Matlab. 


\section{REVIEW OF LITERATURE}

\section{A. Performance Measure}

This describes the ways in which the performance or effectiveness of the different channel estimation techniques was evaluated and requirement of the system with high performance in terms of data rate and capacity, a system that is reliable, less complex, energy efficient and bandwidth efficient. The major figure of merit employed as a measure of comparison in this paper is the BER and MSE versus SNR curve. The various channel estimation techniques were compared based on how accurate the channel is estimated and signal detected, that is how many of the estimated bits are in error. Also, from the BER versus SNR curve, the amount of power required to achieve a particular BER can also be seen, hence used for energy efficiency comparison. In terms of bandwidth efficiency, the methods can also be compared based on the number of pilots required to accurately estimate the channel. Pilots occupy bandwidth, therefore the method that requires lesser number of pilots, and still accurately estimates the channel can be said to be more bandwidth efficient. Also in terms of interpolation, the various interpolation techniques employed can also be compared, and the technique that best interpolates the channel, noted. Taken into consideration also is the system complexity. Some techniques might perform well, but at the expense of system complexity. System complexity can be judged based on the number of mathematical operations required in each technique. The more the mathematical operations (like additions, multiplications, complex matrix inversion etc.) required, the more time it takes to run. This can also mean more power consumption. Therefore the system power consumption can be compared based on the amount of mathematical operations required, and the amount of time taken to execute.

1) Signal to Noise Ratio (SNR): The SNR can be defined as the ratio of the signal power to the noise power. It is a measure used to compare the level of the desired signal strength to the level of the unwanted background noise. It is expressed in decibels $(\mathrm{dB})$. It can be used as a measure of comparison for the energy efficiency of a system, by determining the amount of power required to achieve a desirable BER and MSE [11].

2) Bit Error Rate (BER): The BER is the ratio of the total number of bits received in error to the total number of bits transmitted. These errors could be as a result of interference, noise or distortion. An approximate estimate of the BER is the probability of bit error (or bit error probability). The probability of bit error can be described as the expectation value of the BER. The BER can be affected by factors like the transmission channel, noise, distortion, multipath propagation and fading. To improve the BER, some techniques need to be implemented to checkmate these factors. With respect to the channel, if the channel is accurately estimated, measures (like equalization and pre-coding) can be put in place to combat the negative effects of the channel. Also by applying robust modulation and demodulation techniques, and forward error correction codes, the BER performance can be improved. The BER can be expressed as a function of SNR, which is the normalized carrier to noise ratio, denoted as $E_{b} / N_{0}$, where
TABLE I

Cost of VARious Mathematical Operations [12]

\begin{tabular}{|c|c|}
\hline OPERATION & COST \\
\hline Addition & 1 \\
\hline Multiplication & 10 \\
\hline Division & 40 \\
\hline Square Root & 50 \\
\hline
\end{tabular}

$E_{b}$ represents the energy per bit, and $N_{0}$ is the noise power spectral density. The average BER for the Quadrature phase shift keying (QPSK) can be given as shown in equation (1) [11],

$$
B E R=0.5 \quad\left(1-\sqrt{\frac{S N R(\text { linear })}{1+S N R(\text { linear })}}\right)
$$

3) Mean Squared Error (MSE): The MSE is used to determine the performance of an estimator. The MSE of an estimator can be used to describe and quantify the difference between the actual (true) and estimated data. It is a risk function, and measures the average or expected value of the squares of the errors. The error can be defined as the amount of value by which the estimated value differs from the actual value. In this paper, the MSE can be used to determine how much the estimated channel differs from the actual channel. The MSE can be achieved as shown in equation (2); where $h$ is the actual channel hest is the estimated channel respectively [11].

$$
M S E=E\left|h-h_{e s t}\right|^{2}
$$

4) Bandwidth Efficiency: This describes how efficiently the limited available frequency spectrum is utilized. As a measure of comparison, the pilot-data insertion ratio can determine the bandwidth efficiency. A system that allows more data transmission than pilots can be said to be more bandwidth efficient than that which allows lesser data transmission than pilots.

5) System Complexity and Power Consumption: System complexity can be judged based on the mathematical operations required. Each mathematical operation is assigned a comparative cost, and the total complexity can be given based on this cost. The cost represents the power required to perform the mathematical operations. Hence a technique that has a lower cost can be said to be more energy efficient [12].

Power Efficiency can also be evaluated by the ability to achieve a target BER at minimum power. Power efficiency can be achieved either at the transmitter or at the receiver. As described in [13] power can be consumed at the transmitter by the transmission of data and pilot symbols, while at the receiver, it can be consumed by data acquisition and processing through computational operations. Therefore, to achieve energy efficiency, unnecessary pilot transmission and complex high cost mathematical operations have to be minimised.

\section{Methodology}

The basic steps taken in the modelling and simulation of a wireless communication system, with channel estimation in this paper are described in this section. To run the simulations, 
TABLE II

System Simulation Parameters and VALUES

\begin{tabular}{|c|c|}
\hline SYSTEM PARAMETERS & VALUE \\
\hline Simulation Runs & 100000 \\
\hline Data-Length & 128 \\
\hline Frame-Length & 64 for QPSK \\
\hline SNR Values(dB) & Multipath Channel \\
\hline Channel Type & 5 \\
\hline Number of Channel Taps & 10 \\
\hline Cyclic Prefix & $1: 1,1: 3,1: 7$ and $1: 15$ \\
\hline Pilot-Data Ratio & QPSK \\
\hline Modulation Techniques & SISO, MISO (2X1), MIMO (2X2) \\
\hline Antenna Configurations &
\end{tabular}

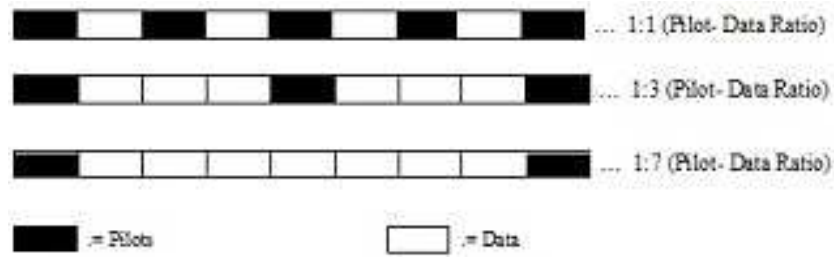

Fig. 1. Pilot Data Insertion Ratio

a transmitter, channel and receiver are required. The figures of merit used are the BER and MSE as a function of the SNR. The BER versus the SNR was plotted on a two dimensional graph using the plot tool in Matlab. Table II shows system initialization parameters employed in this paper.

Steps in the simulation are described below.

Initialization: The first step taken is the initialization of the system parameters. See table 1 . The SNR in dB are established for values between 0 and 30, and later converted to the linear form. The data length and frame length, number of cyclic prefix, are also initialized. The number of transmit and receive antennas are also initialized. The SISO, MISO and MIMO systems were employed.

Transmitter: The transmitter stage is responsible for the modulation and transmission of the signal. After initialization, the data for transmission is generated. In this case, random binary data bits are generated as inputs to be transmitted, since we are employing digital modulation. The generated data can be in vector form, with length equal to the initialized data length of 128. A set of equally spaced pilots are also generated, to be used to estimate the channel. In the comb type, the pilots are not inserted in all subcarriers, but by using a specific pilot-data insertion ratio as shown in Figure 1. Hence interpolation is required in the frequency domain. Due to the importance of interpolation, to estimate the channel at the data subcarriers, various interpolation techniques are compared and analysed, to identify the one with the most optimum performance, with acceptable accuracy, less complexity and less power consumption. Different pilot insertion ratio are analysed and compared, and the minimum amount of pilot required which is sufficient to interpolate and estimate the channel is identified, to allow for more bandwidth efficiency.

As shown in Figure 1, for the pilot-data ratio of $1: 1$, one pilot is inserted for every one data subcarrier. In other words, two neighbouring pilots are used to interpolate for the channel estimate at one data subcarrier. For the pilot-data ratio of 1:3, one pilot is inserted for every three data subcarriers. In other words, two neighbouring pilots are used to interpolate for the channel estimates of three data subcarriers in-between. For the pilot-data ratio of 1:7, one pilot is inserted for every seven data subcarrier. In other words, two neighbouring pilots are used to interpolate for the channel estimate of seven data subcarriers in-between, and as such for pilot-data ratio of 1:15. The digital modulation scheme employed is the QPSK, which has a data rate of 2 bits per symbol. The randomly generated bits are converted and mapped into QPSK symbols (this can be represented by a 2X64 matrix) for modulation. For the SISO, the signals are transmitted through one antenna, but for MISO and MIMO, it is transmitted through a number of transmit and receive antennas to achieve diversity. Also the space tracking clock code (STBC) was employed in the MISO and MIMO, to achieve a better diversity and multiplexing gain.

Channel: Before the transmission, the channel model is taken into consideration. Since it is a wireless mobile system, we assume that the channel is a multipath fading channel with five taps, due to the multiple signal paths as a result of reflections. The multipath channel is generated as complex random normal distribution. For the MISO, two different channels are generated, and both channels send different copies of the signal to the single receive antenna. While for the MIMO (2X2), four different channels are involved, with the two transmit antennas sending to the two receive antenna simultaneously. Considering the fact that a multipath channel can be subjected to destructive interference, a technique should be put in place to combat the interference. Therefore a multi carrier technique like the OFDM is employed. The QPSK symbols are converted into time domain, using the Inverse Fast Fourier transform (IFFT) function in Matlab, and a Cyclic Prefix of 10 is added periodically. This is to maintain orthogonality and enable cyclic convolution of the symbols and the channel in the time domain to be performed. When the cyclic prefix is longer than the channel impulse response (number of channel taps), the channel can be assumed to be free from ICI, thereby resulting in a diagonal channel. The channel is convolved with the OFDM-QPSK symbol. After the convolution, the cyclic prefix, which was initially added for cyclic convolution is removed.

Additive White Gaussian Noise (AWGN): As the signal passes through the channel, it is contaminated by white noise. This can be achieved by the Additive white Gaussian noise (AWGN). The AWGN is generated as normally distributed random numbers, with a mean of zero, and a variance of one. The AWGN vector must have a length equal to the symbol frame length, which is half of the data length since in QPSK.The received OFDM-QPSK symbol is converted back into the frequency domain, using the FFT function in Matlab. After which the AWGN is added. Converting to frequency domain allows easy estimation and equalization operations in the frequency domain.

Receiver: The receiver performs the reverse operations of the transmitter. The noisy signal is received and demodulated at the receiver, and compared to the originally transmitted bits, to determine the number of received bits that are in error.

Channel Estimation: The received signal in frequency domain is multiplied by the inverse of the pilots or known 
transmitted symbol, to get the channel frequency response (channel estimate). After which interpolation is performed to get the channel estimates where pilots are not inserted. After the channel has been estimated, it is compared with the originally generated channel, to calculate the accuracy of the channel estimates. This is analysed by calculating the MSE between the actual channel and the channel estimate, and plotting it for various SNR values.

Equalization: This is performed in other to reverse the effect of the channel on the signal, and enable accurate detection. In this paper, a simple zero forcing equalization is implemented. Multiplying the received signal with the inverse of the channel equalizes the channel and cancels out the effects of the channel on the signal, giving back the originally transmitted signal. Note that this is implemented in the frequency domain. As a basis of comparison, the received signal is equalized using the actual generated channel, assuming perfect channel knowledge. This is compared with the equalization using the estimated channel with different techniques. Equalization using the estimated channel is achieved by multiplying the received signal with the inverse of the channel estimate. The Detected signals using the original channel and the channel estimate are converted back and rearranged in order to match the format of the originally transmitted bits.

Figures of Merit: The detected signal using actual channel equalization and the detected signal from estimated channel Least Square (LS) and Minimum Mean Squared Error (MMSE) are compared with the originally generated signal. An error is counted each time the detected signal bit is not equal to the originally generated signal bit. In this case, the errors are counted for the detected bits from the channel estimates, and the detected bits from perfect channel knowledge. The summation of the number of bits received in error, divided by the total number of transmitted bits, running for each block of simulation gives the BER. The theoretical BER is also computed and plotted, and used for comparison with, and verification of the simulated BER. The MSE is also plotted for various SNR values (0 to 30). The channel frequency response for the actual channel, estimated channel and interpolated channel are also plotted to compare how accurate the channel is estimated and interpolated.

\section{RESUlTS AND ANALYSIS}

\section{A. BER Comparison for MISO MMSE Channel Estimation with Different Pilot-Data Ratio}

For the MISO MMSE channel estimation shown in Figure 2 , it can be seen that the pilot-data ratio of $1: 1,1: 3$, and 1:7 (unlike in the LS where only $1: 1$ and 1:3) is sufficient to interpolate the channel estimate accurately. This can be as a result of the MMSE being able to minimize the error of the LS channel estimates. But with a pilot-data ratio of 1:15 (4 pilots), there is a severe degradation in the BER even with increase in SNR, as a result, the pilot-data ratio of 1:15 is not sufficient (both for LS and MMSE) to interpolate the channel estimate accurately. Therefore, comparing STBC MISO MMSE channel estimation with the STBC MISO LS, it can be said that the MMSE is more bandwidth efficient than the LS. Since it allows

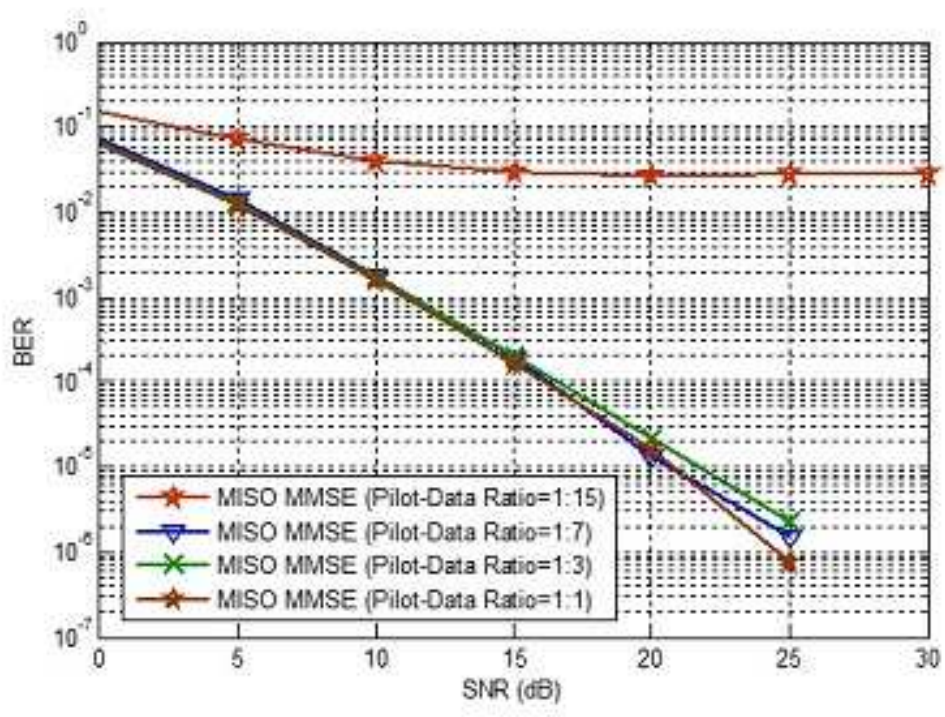

Fig. 2. BER Comparison for MISO MMSE Channel Estimation with Different Pilot-Data Ratio

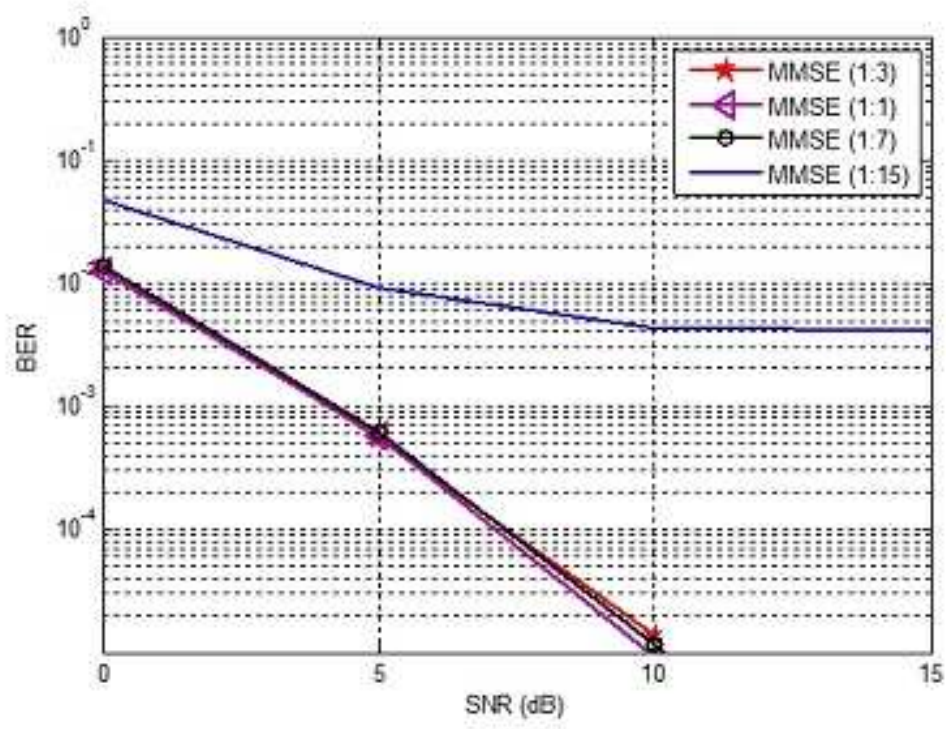

Fig. 3. BER for MIMO (2X2) MMSE Channel Estimation with Different Pilots-Data Ratio

for more data to be transmitted than pilots (with pilot-data ratio of 1:7), and still gives a good performance.

\section{B. BER Comparison for MIMO MMSE Channel Estimation with different Pilot-Data Ratio}

The BER for 2X2 MIMO STBC using MMSE channel estimation is shown in Figure 3. It can be seen from Figure 3 that for pilot-data ratio of $1: 1,1: 3$ and 1:7, the BER performance is very good, achieving an allowable BER threshold of $10^{-3}$ with a low SNR value of about $4 \mathrm{~dB}$. But for pilot-data ratio of $1: 15$, the performance is poor. This further confirms that the pilot-data ratio of $1: 7$ is the optimum pilot insertion ratio for the MMSE, and 1:15 is insufficient to interpolate the channel estimates without errors. Therefore the MMSE (with optimum 


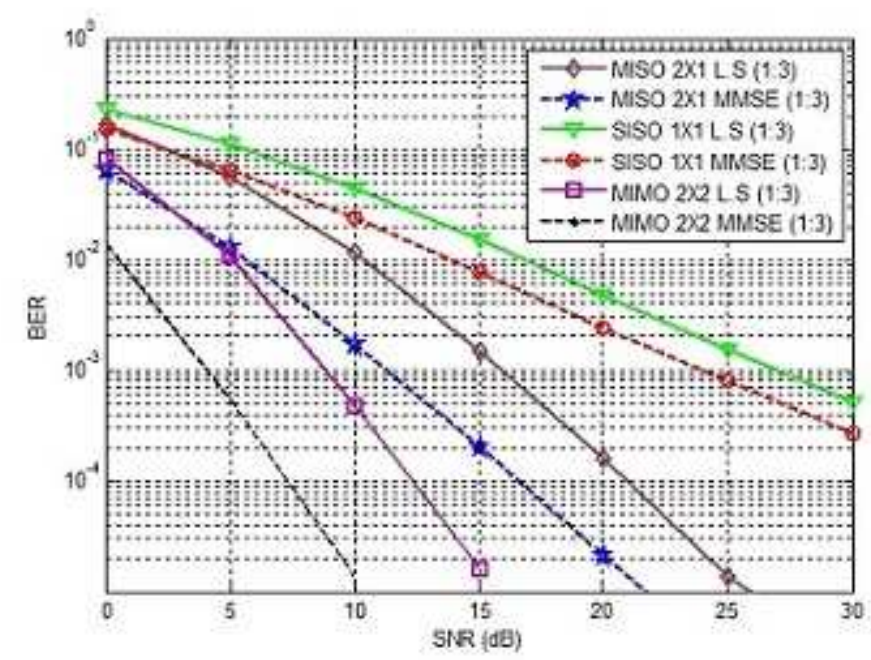

Fig. 4. BER Comparison for SISO, 2X1 MISO STBC and 2X2 MIMO STBC Channel Estimation with Pilot-Data Ratio of 1:3

pilot-data ratio of 1:7) could be said to be more bandwidth efficient than the LS (with optimum pilot-data ratio of 1:3).

\section{BER Comparison for SISO, MISO and MIMO, USING LS and MMSE Channel Estimation}

Figure 4 shows the BER for the SISO, MISO (2X1) and MIMO (2X2) LS and MMSE channel estimation using a pilotdata ratio of 1:3. It can be seen that the MIMO gives the ultimate performance. It can also be seen that the MIMO MMSE achieves a BER of $10^{-3}$ with a low SNR value of about $4 \mathrm{~dB}$, while the MISO MMSE achieves same with an SNR of about $12 \mathrm{~dB}$, and the SISO MMSE achieves the same BER with an SNR of about $24 \mathrm{~dB}$. This can be as a result of the multiplexing and diversity gain exploited by the MIMO and MISO using the STBC technique. Therefore, it can be said that the MIMO is the most energy efficient system, when compared to the MISO and SISO.

\section{BER Comparison for SISO, MISO (2X1) and MIMO (2X2)} $L S$ and MMSE Channel Estimation with Pilot-Data Ratio of $1: 7$

Figure 5 shows the BER for the SISO, MISO (2X1) and MIMO (2X2) LS and MMSE Channel Estimation using a pilot-data ratio of 1:7. It can be seen that the LS channel estimation for SISO, MISO and MIMO could not achieve the BER threshold of $10^{-3}$ with a pilot-data ratio of $1: 7$, giving a degradation in performance even with increase in SNR. It can be seen that the MIMO gives the ultimate performance for both LS and MMSE. This can be as a result of the multiplexing and diversity gain exploited by the MIMO and MISO using the STBC technique. Therefore, it can be said that the MIMO is the most bandwidth and energy efficient system, when compared to the MISO and SISO.

Following the trend of results for the SISO, MISO and MIMO channel estimation, it is obvious that the MMSE outperforms the LS, by achieving a good performance with a

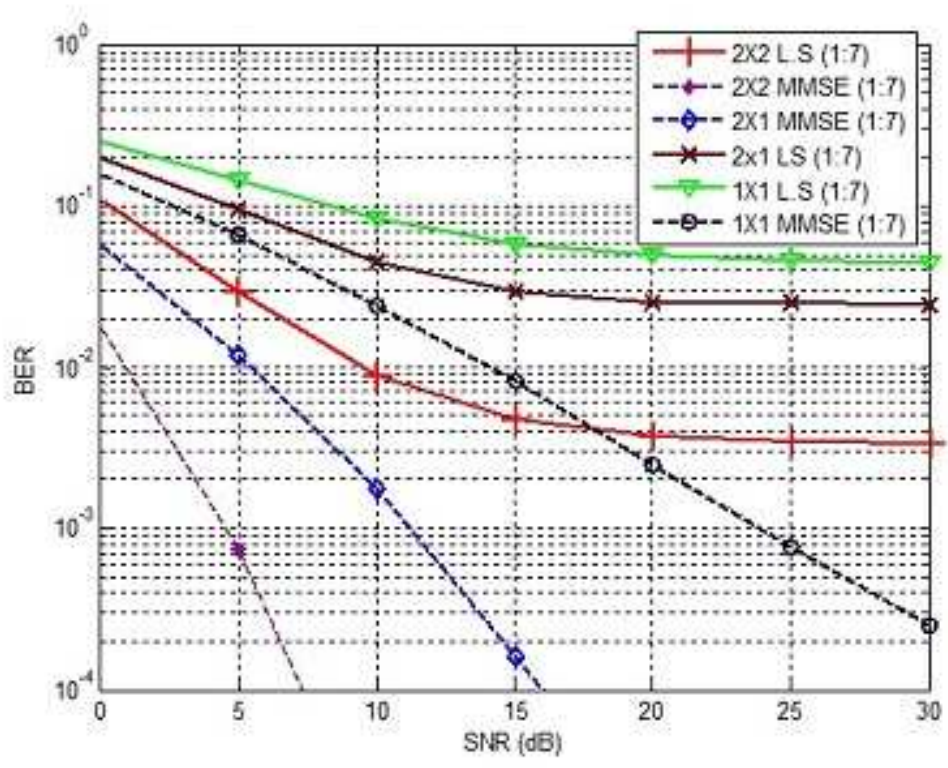

Fig. 5. BER Comparison for SISO, 2X1 MISO STBC and 2X2 MIMO STBC Channel Estimation with Pilot-Data Ratio of 1:7

more bandwidth efficient pilot-data ratio of 1:7. In other words, it is observed that; The LS gave a good BER performance with pilot-data ratio of $1: 1$ and 1:3. The MMSE gave a good BER performance with pilot-data ratio of $1: 1,1: 3$ and 1:7. As a result, the MMSE can be used to achieve a more bandwidth efficient system, achieving good performance even with a pilot-data ratio of 1:7, allowing for the transmission of more data. Although, the MMSE is more computationally complex. A trade-off between performance, bandwidth efficiency and system complexity is observed. Comparing the cost of operation for the MMSE and the LS, the MMSE has a higher cost of operation than the LS which requires only a division or matrix inversion. Therefore, MMSE could require more power consumption.

\section{CONCLUSION}

In this paper, various channel estimation, interpolation and equalization techniques are compared, and the technique with the optimum performance is determined. The comparison is done using the SISO, MISO-STBC, and MIMO-STBC antenna configurations and the difference in performance is shown. The BER and MSE as a function of SNR is the employed Figure of merit. The simulated values are compared with theoretical values, and the actual channel is compared with the estimated channel as a validation standard. The results are generated and presented based on modelling and simulations using Matlab Simulation Package. The LS estimation and MMSE channel estimation techniques are compared. The LS is computationally less complex because of the fewer mathematical operations required, than the MMSE which has more computational complexity. The LS gives a good MSE and BER performance, but requires more SNR (transmit power) to achieve the same performance as the MMSE. The MMSE on the other hand is more resistance to noise than the LS, and 
gives a better performance than the LS. On system complexity and operational cost, the MMSE requires a higher operational cost than the LS. The MMSE also requires a prior knowledge of the noise variance. The different pilot-data insertions ratios are examined, the minimum amount of pilots that are sufficient to accurately interpolate the channel estimates are determined. Using a pilot-data ratio of 1:1 is bandwidth inefficient, because it gives similar performance with a pilot-data ratio of $1: 3$. For the LS, the optimum pilot data ratio is $1: 3$, because with pilot-data ratio of $1: 7$, the LS degrade in performance. The MMSE on the other hand is able to minimize the errors of the LS, by giving a good performance with a pilot-data ratio of 1:7. Therefore the MMSE is more bandwidth efficient than the LS. Channel estimation in SISO, MISO and MIMO are compared. Using the STBC, diversity and multiplexing gain is achieved in the MISO and MIMO. The MIMO is a more energy efficient technique, achieving a good BER performance at lower transmit SNR, when compared to the MISO and SISO which requires higher SNR to achieve same BER performance. The MIMO gives the optimum performance, followed by the MISO and SISO. This is as a result of the diversity and multiplexing gain experienced in the multiple antenna techniques using the STBC.

\section{REFERENCES}

[1] N. Chilamkurti, S. Zeadally and F. Mentiplay, Green Networking for Major Components of Information Communication Technology Systems,
EURASIP Journal on Wireless Communications and Networking, vol. 2009, Article ID 656785.

[2] K.-L. Du and M. N. S. Swamy, Wireless Communication Systems, Cambridge University Press, New York, 2010.

[3] S. Coleri, M. Ergen, A. Puri, and A. Bahai, Channel Estimation Techniques Based on Pilot Arrangement in OFDM Systems IEEE Trans. Broadcasting, vol. 48, no. 3, Sept. 2002, pp 223-229.

[4] M. H. Hsieh and C. H. Wei, Channel Estimation for OFDM Systems Based on Comb-Type Pilot Arrangement in Frequency Selective Fading Channels IEEE Trans. Consumer Electronics, vol. 44, no. 1, Feb. 1998 pp. 217-225.

[5] K. Kwak et al, New OFDM Channel Estimation with Dual-ICI Cancellation in Highly Mobile Channel, IEEE Trans. Wireless Communications, vol. 9 , no. 10 , Oct. 2010 , p. 3156.

[6] $\mathrm{H}$. Yang, A road to future broadband wireless access: MIMO-OFDM based air interface, IEEE Commun. Mag., vol. 43, no. 1, Jan. 2005, pp. 53-60.

[7] H. Sampath, et al, A fourth-generation MIMO-OFDM broadband wireless system: design, performance and Field trial results, IEEE Communications Magazine, No. 9, Sep. 2002, pp. 143-149.

[8] A. Sibille, C. Oestges, and A. Zanella, MIMO from Theory to Implementation, Academy Press, Burlington, USA, 2011.

[9] G. J. Foschini and M. J. Gans, On Limits Of Wireless Communicationsin A Fading Environment When Using Multiple Antennas, Wireless Pers.Commun.,vol. 6, no. 3, Mar. 1998, pp. 311-335.

[10] Y. S. Cho et al, MIMO-OFDM Wireless Communications with MATLAB, IEEE Press, John Wiley and Sons (Asia) Pte Ltd. 2010

[11] T. S. Rappaport, Wireless Communications: Principles and Practice, Second Edition, Prentice Hall, 2002.

[12] J. P. Javaudin, et al,An OFDM Evolution for the UMTS High Speed Downlink Packet Access, France Telecom R and D, DMR/DDH Laboratory, Wireless Technology Labs, Nortel Networks, Station C, Ottawa, Canada.

[13] S. Yatawatta, A. P. Petropulu, and C. J. Graff, Energy-Efficient Channel Estimation in MIMO Systems, EURASIP Journal on Wireless Communications and Networking, vol. 2006, Article ID 27694, pp 111 